\title{
Audit of turn-around times in a microbiology laboratory
}

\author{
S Rogers, M J Bywater, D S Reeves
}

\begin{abstract}
To determine the turn-around time in a microbiology laboratory a survey form was designed to collect data on the origin and type of specimen and the dates and times when (i) the sample was taken; (ii) the specimen was received in the laboratory; (iii) the report was signed by the microbiologist; (iv) the report was sorted by the laboratory clerical staff; and (v) the final report was received on the ward. The cumulative time from sampling to a result arriving on the ward was $51 \cdot 5$ (SD 28.6) hours.
\end{abstract}

The survey is a simple method of determining turn-around time and highlighted minor deficiencies in the service which were easily rectified.

Providing clinicians with accurate results is only one of the many functions of a pathology department. In microbiology, audit in the form of voluntary participation in internal and external quality assurance schemes has been operating in Britain for many years, ${ }^{1}$ but very little has been done in terms of a broader assessment schemes have been developed in the United States for examining other areas of laboratories' performance, such as turnaround time and the utilisation of laboratories. ${ }^{2}$ We were unaware of any previously published survey of turn-around time in microbiology in this country so we set one up using what we hoped would be a simple system.

Papartment of Hospital, Westbury On Trym, Bristol, BS10 5NB

M J Bywater

D S Reeves

Department of

Pathology, University of Sheffield Medical

School, Beech Hill

Road, Sheffield

S10 2RX

S Rogers

Correspondence to:

Dr S Rogers

Accepted for publication

6 September 1990

Total cumulative mean (SD) times in hours with standard deviation

\begin{tabular}{llll}
\hline Day & Urine samples only & Excluding urine samples & Total samples \\
\hline Monday & $29 \cdot 9(8 \cdot 9) \mathrm{n}=17$ & $40 \cdot 0(17 \cdot 2) \mathrm{n}=13$ & $34 \cdot 2(13 \cdot 9) \mathrm{n}=30$ \\
Tuesday & $38 \cdot 0(19 \cdot 1) \mathrm{n}=14$ & $51 \cdot 7(18 \cdot 2) \mathrm{n}=6$ & $42 \cdot 19(1 \cdot 4) \mathrm{n}=20$ \\
Wednesday & $28 \cdot 7(7 \cdot 5) \mathrm{n}=16$ & $70 \cdot 2(41 \cdot 3) \mathrm{n}=6$ & $40 \cdot 0(28 \cdot 4) \mathrm{n}=22$ \\
Thursday & $57 \cdot 0(31 \cdot 2) \mathrm{n}=14$ & $89 \cdot 4(33 \cdot 9) \mathrm{n}=6$ & $66 \cdot 7(34 \cdot 7) \mathrm{n}=20$ \\
Friday & $59 \cdot 4(29 \cdot 7) \mathrm{n}=19$ & $83 \cdot 6(16 \cdot 4) \mathrm{n}=10$ & $67 \cdot 8(28 \cdot 1) \mathrm{n}=29$ \\
Saturday & $67 \cdot 8(10 \cdot 3) \mathrm{n}=3$ & $59 \cdot 7(28 \cdot 2) \mathrm{n}=8$ & $61 \cdot 9(24 \cdot 4) \mathrm{n}=11$ \\
Sunday & $48 \cdot 4(24 \cdot 7) \mathrm{n}=5$ & $79 \cdot 8(20 \cdot 4) \mathrm{n}=3$ & $60 \cdot 1(27 \cdot 1) \mathrm{n}=8$ \\
\hline
\end{tabular}

\section{Methods}

The survey form was designed to collect data on the origin and type of specimen and the dates and times when: (1) The sample was taken; (2) the specimen was received in the laboratory; (3) the report was signed out by the microbiologist; (4) the report was sorted by the laboratory clerical staff; (5) the final report was received on the ward. appraisal of laboratory functions. Quality

All hospital staff involved were informed of the survey by letter. Three hundred and fifty survey forms were distributed to eight target areas in the hospital at different distances on the site from the laboratory. The form accompanied the request and report forms at all stages through the process and each of the five sections was completed with the date and time by a doctor, MLSO, or clerk.

The survey was conducted over 11 weeks, including two bank holidays. Of 180 survey forms returned 40 were unsuitable for inclusion due to incomplete information. The findings were entered into a microcomputer database for analysis.

\section{Results}

Most completed forms (62\%) related to urine specimens. As might be expected, the longest interval was that between the specimen arriving in the laboratory and being signed out by the microbiologist, with a mean time of $36 \cdot 1$ hours (SD 22.0). The cumulative mean time (CMT) from sampling to a result arriving on the ward was 51.5 hours (SD 28.6). This time varied with the distance from the laboratory, being 39.1 (SD 31.4) for the nearest ward and 68.1 (SD 31.3) for the most distant ward. The latter provide long-stay, non-acute services, and the apparent delay may represent failure to acknowledge receipt of the report due to the part-time ward clerical staff being off duty when the results were delivered.

Analysis by day of the week shows that the total CMT increases at the end of the working week and at weekends (table).

\section{Comment}

Our survey represents a simple approach to evaluating turn-around time of our laboratory. The main difficulties which we found related to failure to complete the survey forms correctly. There were also some communication problems, particularly in relation to the bank holidays when the different portering staff on duty were unaware of the survey, with the result that the data were less complete for these days. Data were available, however, for two urine samples, with total CMTs of 28.8 hours and 28.3 hours, which suggests that services were not significantly disrupted during this period.

This exercise provided us with important information by highlighting weaknesses in our 
system at which we were able to target attempts at improvements. We would like to point out that we have a policy for telephoning all important results to the clinicians directly, so by-passing the written report.

In future, the computerisation of the microbiology service with terminals in all clinical areas will lead to a more efficient turnaround time.

1 Pennington GW. Laboratory accreditation and audit in pathology. The maintenance of standards of pathology in
Britain: the current position. J Clin Pathol 1989;42:561-2.

2 Hoffman G. Laboratory accreditation and audit in pathology: Medical audit in the United States. J Clin Pathol 1989;42:562.

\title{
Comparative yield of Salmonella typhi from blood and bone marrow cultures in patients with fever of unknown origin
}

\author{
B J Farooqui, $M$ Khurshid, $M$ K Ashfaq, $M$ Ata Khan
}

\begin{abstract}
Over three years, a comparative study on 100 selected patients with fever of unknown origin was undertaken to determine the yield of Salmonella typhi from their blood and bone marrow cultures. The results indicate that in patients who had an infection with $S$ typhi the organism was isolated from the bone marrow in all of them and from the blood in only $66 \%$. This suggests that bone marrow cultures may be attempted when blood cultures are negative for bacterial growth after three to four days of incubation.
\end{abstract}

Infection with Salmonella typhi is one of the commonest causes of typhoid fever in Karachi, Pakistan. Over three years, we processed 21356 blood samples from patients suspected of typhoid fever. The average isolation rate for Salmonella from these blood cultures was $5 \cdot 15 \%$. Further subtyping of these Salmonella species showed an incidence of $S$ typhi $83 \%$, $S$ paratyphi $A \quad 11 \cdot 8 \%, S$ paratyphi $B \quad 4 \cdot 1 \%$ and $S$ paratyphi $C 0.9 \% .{ }^{1}$ Definitive diagnosis is made on the basis of isolation of the organism from blood. ${ }^{2}$ Without antibiotics blood cultures are positive in over $80 \%$ of the patients seen in the first week of illness. ${ }^{3}$ Twenty to $30 \%$ of untreated patients have positive blood cultures as late as the third week of illness.

Antimicrobial treatment diminishes the possibility of recovery of the organism in blood. In patients who have been treated cultures of supplementary sites such as bone marrow may be a better source of organisms than blood. ${ }^{4}$ We conducted a study on 100 selected patients whose blood and bone marrow were cultured for $S$ typhi to determine the yield of $S$ typhi from these two sites.

\section{Methods}

Blood and bone marrow samples were collected from 100 patients who had fever of unknown origin. Each sample was collected in two blood culture bottles containing brain heart infusion and thioglycollate broth, respectively.

About $5 \mathrm{ml}$ of venous blood was inoculated in each bottle containing $45 \mathrm{ml}$ of broth; similarly, $0.5 \mathrm{ml}-1.0 \mathrm{ml}$ of bone marrow was collected in each bottle containing $45 \mathrm{ml}$ of broth. Bottles were sent immediately to the laboratory and incubated at $37^{\circ} \mathrm{C}$ for seven days. Each bottle was examined daily and subcultured on to blood agar and MacConkey's media after 24, 48, and 72 hours, and on the seventh day of incubation.

Non-lactosing fermenting colonies from MacConkey's medium were tested for $S$ typhi by slide agglutination test with specific antisera. ${ }^{56}$ Biochemical tests were performed by using API 20E strips.

\section{Results}

The samples of blood and bone marrow were processed at The Aga Khan University Hospital's diagnostic laboratory. $S$ typhi was isolated from the blood $(n=58)$ or bone marrow $(n=88)$. The blood and bone marrow cultures of 12 patients showed no growth for any bacteria.

Thirty blood samples from 88 patients (whose bone marrow were positive) showed no bacterial growth. 